\title{
ESTUDO DO EFEITO DA ADIÇÃO DE DIFERENTES TEORES DE ARGILAS MONTMORILONITA E MICA MUSCOVITA NO COMPORTAMENTO PROTETIVO DE TINTAS EM PÓ BASE EPÓXI NA APLICADAS SOBRE AÇO AISI 1008
}

\author{
Patrícia da Costa Ferreira' \\ Lilian Vanessa Rossa Beltrami ' \\ Diego Piazza' \\ Sandra Raquel Kunst ' \\ Ademir Jozé Zattera '
}

\section{Resumo}

O presente trabalho apresenta a elaboração de nanocompósitos poliméricos de tinta em pó, à base de resina epoxídica e diferentes teores de argilas montmorilonita e mica muscovita. Os nanocompósitos foram obtidos no estado fundido em extrusora duplarrosca co-rotante e foram analisados por ensaios de aderência, perfilometria, ângulo de contato, espectroscopia de impedância eletroquímica e névoa salina. Os resultados indicam que a rugosidade superficial aumentou com o aumento das concentrações de argilas. Em geral a grande maioria das amostras com argilas apresentaram melhora nas propriedades protetivas, quando comparadas com as amostras sem adição de argila.

Palavras-chave: Corrosão; Montmorilonita: Mica muscovita.

\section{STUDY OF THE EFFECT OF THE ADDITION OF DIFFERENT LEVELS OF MONTMORILLONITE AND MICA MUSCOVITE CLAYS ON THE PROTECTIVE BEHAVIOR OF EPOXY BASE POWDER PAINTS APPLIED ON AISI 1008 STEEL}

\begin{abstract}
The present work presents the elaboration of polymer nanocomposites of powder paint, based on epoxy resin and different levels of montmorillonite and muscovite mica clays. The nanocomposites were obtained in the molten state in a co-rotating double-screw extruder. The nanocomposites properties were analyzed by adhesion, profilometry, contact angle, electrochemical impedance spectroscopy and salt fog tests. The results indicate that the surface roughness increased with increasing concentrations of clays. In general, the majority of samples with clays showed improvement in the protective properties when compared to the samples without addition of clay.
\end{abstract}

Keywords: Corrosion; Montmorillonite; Muscovite mica.

\section{INTRODUÇÃO}

Revestimentos orgânicos, como tintas, são amplamente utilizados sobre a superfície de materiais metálicos, com a finalidade estética e protetiva. Atualmente, as tintas em pó vêm sendo muito utilizadas devido a sua facilidade de aplicação e armazenamento, além de menor desperdício e da não utilização de solventes nocivos à saúde humana. Pesquisas mostram que nanotecnologias associadas às tintas apresentam como resultado novos revestimentos compósitos com excelentes propriedades $[1,2]$.

Nanocompósitos poliméricos são definidos como uma classe de materiais contendo uma matriz polimérica reforçada com cargas que apresentem ao menos uma das dimensões nanométrica (nanocargas), como minerais, fibras e argilas, por exemplo [3-5].

I Universidade de Caxias do Sul - UCS, Caxias do Sul, RS, Brasil 
O uso de argilomineirais como montmorilonita (MMT) e mica muscovita (Mica) como cargas em compósitos poliméricos tem sido motivo estudos para desenvolvimento de novos nanocompósitos [3-9]. As argilas montmorilonita (MMT), têm sido utilizadas como cargas para a obtenção de revestimentos nanoestruturados, proporcionando melhorias significativas no desempenho protetivo frente à corrosão e propriedades de barreira em revestimentos orgânicos [I-6].

Contudo, a concentração destas nanocargas aos polímeros é um fator determinante nas propriedades finais do nanocompósito. Estudos mostraram que a adição de nanocargas em baixa concentração (inferior a $10 \%$ em massa) proporciona uma melhora nas propriedades da matriz tais como, propriedades mecânicas, de barreira e principalmente impermeabilidade e inflamabilidade, dentre outras $[8,10-12]$.

Em relação ao método de preparação do nanocompósitos contendo nanocargas à base de argilominerias, destaca-se os métodos de esfoliação-adsorção [13], intercalação por polimerização in-situ [14] ou intercalação no estado fundido [15]. A técnica de intercalação no estado fundido tem sido preferida devido às inúmeras vantagens como ausência de solventes e capacidade de produção elevada [15].

Diante de tudo isso, destaca-se o grande potencial da utilização de argilominerais como nanocargas em resinas termorrígidas, visando o desenvolvimento de nanocompósitos, como tintas em pó. Com isso, espera-se que estas novas tintas em pó apresentem melhoras propriedades mecânicas, de barreira e de resistência à degradação [16].

Com isso, este trabalho apresenta um estudo comparativo entre a incorporação de diferentes tipos de nanocargas argilominerais (montmorilonita e mica moscovita) a uma formulação de tinta em pó base epóxi. O objetivo do presente trabalho é avaliar a influência da concentração das nanocargas em diferentes proporções (2, 4 e $6 \%$ m/m) na obtenção de nanocompósitos poliméricos, avaliando suas propriedades morfológicas e protetivas.

\section{MATERIAIS E MÉTODOS}

\section{I Materiais}

Neste trabalho utilizou-se as argilas montmorilonita (MMT), dos tipos Cloisite ${ }^{\circledR}$ 30B (MMT 30B) e Cloisite ${ }^{\circledR}$ I5A (MMT I5A), fornecidas pela Southern Clay Products, e mica muscovita (Mica), fornecida pela Lamil Lage Minérios.

A resina epóxi comercial utilizada é da marca Araldite GT7004 ES, fornecida pela Hunstman. Para a formação da tinta utilizou-se o agente de cura I-otolibiguanida derivado da diciandiamida (Casamid 710 Oiled), fornecido pela Thomas Swan \& Co. Ltd., o agente alastrante Resiflow PV-60, fornecido pela Estron Chemical Inc., a benzoína, fornecida pela Datiquim Produtos Químicos Ltda. Como substrato metélico, utilizou-se o aço carbono AISI 1008 , com dimensões de $70 \times 120 \times 0,75 \mathrm{~mm}$.

\section{I.I Obtenção do revestimento}

Para obtenção dos nanocompósitos de tinta epóxi em pó (TE) foram adicionadas nanocargas argilomineirais, nas proporções de 2, 4 e $6 \%(\mathrm{~m} / \mathrm{m})$ à resina epóxi em uma extrusora dupla-rosca co-rotante, modelo MH-COR-LAB, com diâmetro de rosca de $20 \mathrm{~mm}$ e relação L/D de 32, fabricado pela MH Equipamentos Ltda.

A extrusora possui oito zonas de aquecimento, onde a zona de alimentação possui temperatura de alimentação de $70^{\circ} \mathrm{C}$ e todas as outras em $90^{\circ} \mathrm{C}$, com velocidade de processamento de $200 \mathrm{rpm}$, com taxa de alimentação de $5,5 \mathrm{~kg} \cdot \mathrm{h}^{-1}$. Após a extrusão, os materiais foram moidos em um moinho de facas, da marca Cadence MDR 30I, e posteriormente peneirados em peneira 200 mesh.

As amostras de aço carbono, antes da aplicação dos revestimentos orgânicos, foram previamente lixados manualmente, utilizando-se lixas de granulometria 320,400 e 600 , e posteriormente submetidos ao tratamento de fosfatização, com fosfato de zinco (Klintex Insumos Industriais Ltda) passando pelas etapas de desengraxe, refinação, fosfatização, passivação e lavagem.

As tintas epóxi em pó foram aplicados sobre os substratos de aço carbono por pistola de pulverização eletrostática, da marca TCA ECO Tecnoavance. A cura foi realizada em forno de circulação de ar, a $200^{\circ} \mathrm{C}$ por 20 minutos. A nomenclatura adotada para identificação das amostra está descrita na Tabela I.

\subsection{Caracterização da Tinta em Pó Aplicada Sobre - Aço Carbono}

A investigação do perfil de rugosidade da superfície das amostras estudadas foi realizada a partir do ensaio de perfilometria, utilizado-se um perfilômetro Mitutoyo, modelo Surftest SJ-30 I.

Para o teste de ângulo de contato as amostras foram submetidas ao método da gota séssil, no Laboratório de Pesquisa em Química de Materiais (LPQM) da Universidade de Caxias do Sul (UCS). Com uma seringa B-D Yale 3D deposita-se uma gota sobre o revestimento e observa-se o ângulo formado por meio de fotos utilizando lente de baixo aumento com a máquina Lumix FZ40 Panasonic DMC-FZ40 24x. O ângulo de contato foi determinado utilizando o software de análise de imagem e as medidas realizadas pelo Software Surftens 3.0.

A espectroscopia de impedância eletroquímica foi realizada utilizando uma célula de três eletrodos, posicionada no interior de uma gaiola de Faraday. Utilizou-se como eletrodo de trabalho as amostras a serem testadas; eletrodo de calomelano saturado (ECS) como eletrodo de referência e uma espiral de platina como contra eletrodo. As medidas de EIE foram realizadas expondo uma área de $78 \mathrm{~mm}^{2}$ da amostra em uma solução de $3,5 \%(\mathrm{~m} / \mathrm{v})$ de $\mathrm{NaCl}$. Os dados de EIE foram obtidos utilizando um potenciostato/galvanostato IviumStat da Ivium Technologies, auxiliado pelo software 
Tabela I. Nomenclatura adotada para identificação das amostras estudadas

\begin{tabular}{|c|c|}
\hline Nomenclatura & Descrição \\
\hline TE / 0 & Tinta epóxi sem adição de argila \\
\hline $\mathrm{TE} / 2 / 30 \mathrm{~B}$ & Tinta epóxi com adição de $2 \%(\mathrm{~m} / \mathrm{m})$ de argila Cloisite ${ }^{\circledR} 30 \mathrm{~B}$ \\
\hline TE / 4 / 30B & Tinta epóxi com adição de $4 \%(\mathrm{~m} / \mathrm{m})$ de argila Cloisite ${ }^{\circledR}$ 30B \\
\hline $\mathrm{TE} / 6 / 30 \mathrm{~B}$ & Tinta epóxi com adição de $6 \%(\mathrm{~m} / \mathrm{m})$ de argila Cloisite ${ }^{\circledR} 30 \mathrm{~B}$ \\
\hline TE / 2 / I5A & Tinta epóxi com adição de $2 \%(\mathrm{~m} / \mathrm{m})$ de argila Cloisite ${ }^{\circledR}$ I5A \\
\hline TE / 4 / I5A & Tinta epóxi com adição de $4 \%(\mathrm{~m} / \mathrm{m})$ de argila Cloisite ${ }^{\circledR}$ I5A \\
\hline TE / $6 / 15 \mathrm{~A}$ & Tinta epóxi com adição de $6 \%(\mathrm{~m} / \mathrm{m})$ de argila Cloisite ${ }^{\circledR}$ I5A \\
\hline TE / 2 / Mica & Tinta epóxi com adição de $2 \%(\mathrm{~m} / \mathrm{m})$ de argila Mica moscovita \\
\hline TE / 4 / Mica & Tinta epóxi com adição de $4 \%(\mathrm{~m} / \mathrm{m})$ de argila Mica moscovita \\
\hline TE / 6 / Mica & Tinta epóxi com adição de $6 \%(\mathrm{~m} / \mathrm{m})$ de argila Mica moscovita \\
\hline
\end{tabular}

IviumSoft, aplicando uma faixa de frequência de $10^{5} \mathrm{a} 0, \mathrm{I} \mathrm{Hz}$, e uma amplitude de perturbação senoidal de $10 \mathrm{mV}$ em torno do potencial de corrosão. As medidas foram realizadas como função do tempo de imersão.

As amostras foram expostas a névoa salina conforme Norma ASTM BI 17 (2007), por 504 horas em uma câmara fechada, marca Bass Equipamentos modelo USX-6000/2009, fixadas na câmara em apoios com ângulos entre 15 e $30^{\circ} \mathrm{em}$ relação à vertical. $\mathrm{O}$ ensaio foi realizado em triplicata $\mathrm{e}$ conduzido conforme as seguintes condições: pressão do ejetor de 0,7 a I, $7 \mathrm{kgf.cm}{ }^{-2}$; temperatura da câmara de $35^{\circ} \mathrm{C} \pm 2^{\circ} \mathrm{C}$; solução de cloreto de sódio $5 \% \pm 1 \%$; faixa de $\mathrm{pH}$ entre 6,5 a 7,2 e volume de névoa coletada de I a $2 \mathrm{~mL} . \mathrm{h}^{-1}$.

A fim de avaliar a migração subcutânea das amostras submetidas ao ensaio de névoa salina, uma incisão em " $X$ " foi realizada na superfície das amostras, conforme Norma ASTM DI654 (2008). As medições foram realizadas em 10 pontos ao longo de cada incisão por amostra.

\section{RESULTADOS E DISCUSSÕES}

A rugosidade média superficial das amostras estudadas é apresentada na Tabela I. Observa-se que a rugosidade média aumentou gradativamente ao aumento do percentual de argila, sendo que, este aumento foi mais acentuado para as amostras contendo MMT (30B e I5A). Este resultado pode estar associado à aglomeração da argila MMT o que provoca um aumento da rugosidade final da tinta. Segundo alguns autores $[17,18]$, a incorporação de teores de argila superiores a $1 \%$ em peso, pode promover a formação de aglomerados, devido à alta energia superficial das argilas, impedindo a sua dispersão na matriz polimérica.

O baixo valor de rugosidade apresentado para a amostra TE/0 é devido ao agente de cura derivado da diciandiamida, na qual envolve reações de adição tanto com os grupos glicidila quanto com os grupos hidroxila e, simultaneamente, catalisa a reação entre dois grupos glicidila. Este catalisador é muito utilizado em agentes de cura para tinta em base pó epóxi pois apresenta uma excelente reatividade e obtêm-se revestimentos com excelentes propriedades em termos de nivelamento e brilho. Com a adição de diciandiamida diminui-se problemas decorrentes da falta de homogeneidade apresentando um revestimento com boa aparência de revestimento e flow [19].

A Tabela I também apresenta os valores médios dos ângulos de contato formados entre uma gota de água e a superfície das amostras estudadas. Sabe-se que a molhabilidade de uma superfície é influenciada pela sua natureza e heterogeneidades químicas, estando associada também a sua textura superficial (topografia e rugosidade) [20]. Contudo, mesmo com rugosidades médias superficiais distintas, o ângulo de contato médio das amostras não apresentou variações significativas, quando comparadas à amostra sem argila $(\mathrm{TE} / 0)$. Este resultado indica que a adição das argilas MMT e Mica, em qualquer concentração, não alterou a energia superficial das amostras, mantendo as superfícies hidrofílicas. Isso pode ter ocorrido devido as partículas de argila estarem recobertas pela resina, não alterando significativamente a superfícies.

\section{I Ensaio de Névoa Salina}

A Figura I apresenta o aspecto das amostras após 504 horas de exposição ao ensaio de névoa salina. Durante o ensaio, observou-se que as amostras apresentaram corrosão vermelha na incisão após 24 horas de exposição à névoa salina. Ao longo do ensaio, os produtos de corrosão foram aumentando e escorrendo pela superfície da amostra.

Todas as amostras apresentaram pontos de empolamento de baixo grau no decorrer do ensaio. A presença de bolhas pode facilitar a penetração da água, oxigênio e íons através do revestimento, resultando em uma corrosão localizada [19,2I]. Bagherzadeh et al. [22] relatou um resultado em seu estudo que após 500 horas de exposição à névoa salina, todos as amostras revestidas com tinta epóxi reforçadas com argila apresentaram formação de bolhas e pontos de corrosão na região próxima à incisão. Segundo os autores, este comportamento ocorreu pela difusão da água sob através do revestimento, devido a presença dos defeitos.

Ao final do ensaio, após 504 horas, observou-se que as amostras TE/6/30B e TE/6/I5A (Figura Id e Ig, 

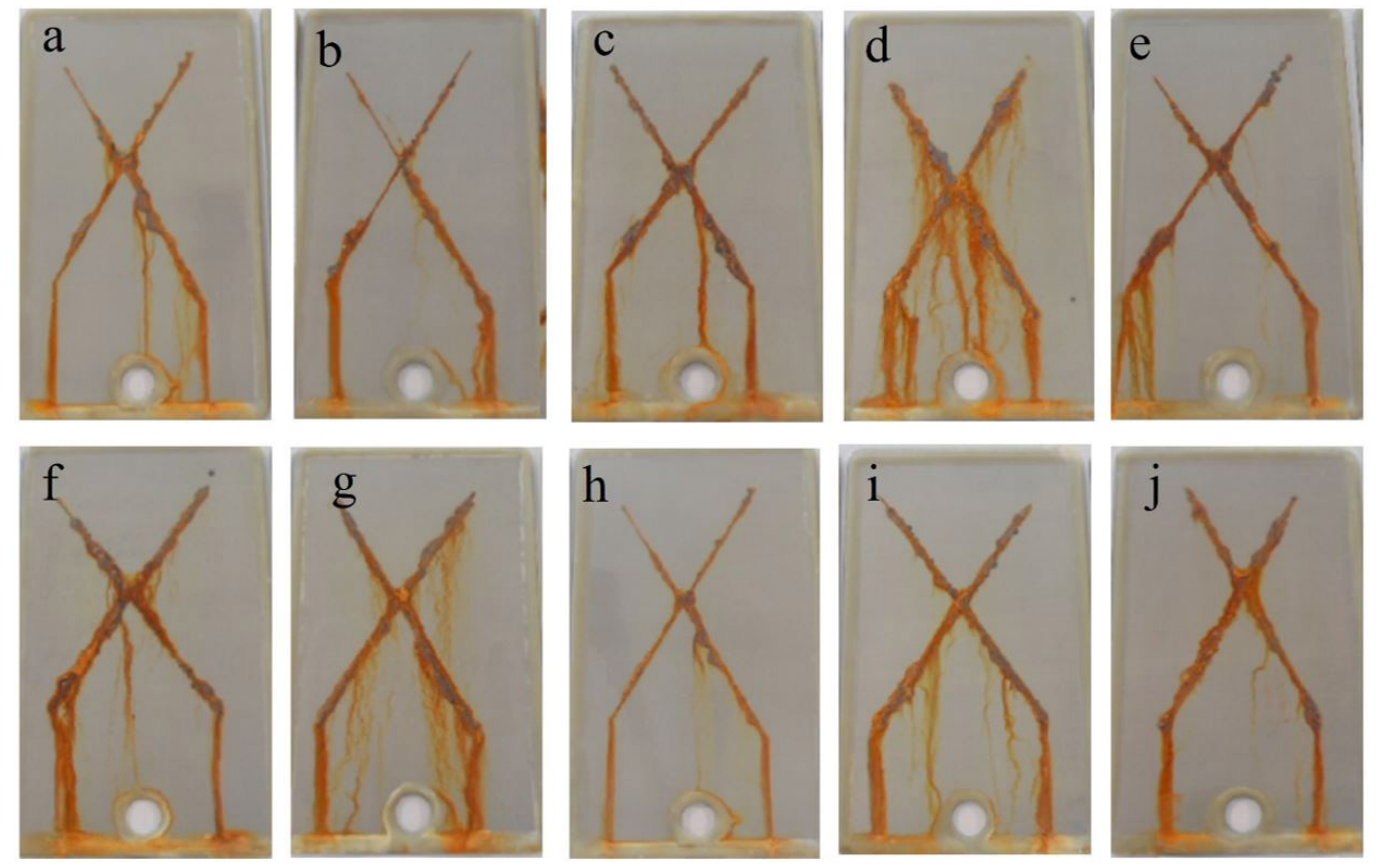

Figura I. Aspecto das amostras dos substratos metálicos revestidos com as tintas em pó base resina epóxi após 504 horas de exposição ao ensaio de névoa salina: (a) TE/0, (b) TE/2/30B, (c) TE/4/30B, (d) TE/6/30B, (e) TE/2/I5A, (f) TE/4/I5A, (g) TE/6/I5A, (h) TE/2/Mica, (i) TE/4/Mica e (j) TE/6/Mica.

Tabela 2. Rugosidade e molhabilidade dos revestimentos estudados

\begin{tabular}{ccc}
\hline Amostra & $\begin{array}{c}\text { Rugosidade média } \\
(\boldsymbol{\mu} \mathbf{m})\end{array}$ & $\begin{array}{c}\text { Ângulo de contato } \\
\left({ }^{\circ}\right)\end{array}$ \\
\hline $\mathrm{TE} / 0$ & $0, \mathrm{I} \pm 0, \mathrm{I}$ & $7 \mathrm{I} \pm 2$ \\
$\mathrm{TE} / 2$ / 30B & $0,2 \pm 0,0$ & $69 \pm \mathrm{I}$ \\
$\mathrm{TE} / 4$ / 30B & $1,0 \pm 0, \mathrm{I}$ & $7 \mathrm{I} \pm 2$ \\
$\mathrm{TE} / 6$ / 30B & $4,7 \pm 0,2$ & $70 \pm \mathrm{I}$ \\
$\mathrm{TE} / 2$ / I5A & $0,1 \pm 0,0$ & $69 \pm \mathrm{I}$ \\
$\mathrm{TE} / 4$ / I5A & $\mathrm{I}, 4 \pm 0,5$ & $70 \pm \mathrm{I}$ \\
$\mathrm{TE} / 6$ / I5A & $6,5 \pm 0, \mathrm{I}$ & $69 \pm \mathrm{I}$ \\
$\mathrm{TE} / 2$ / Mica & $0,1 \pm 0, \mathrm{I}$ & $70 \pm 1$ \\
$\mathrm{TE} / 4$ / Mica & $0,2 \pm 0, \mathrm{I}$ & $70 \pm \mathrm{I}$ \\
$\mathrm{TE} / 6 /$ Mica & $0,3 \pm 0, \mathrm{I}$ & $69 \pm \mathrm{I}$ \\
\hline
\end{tabular}

respectivamente) apresentaram corrosão e empolamento mais intensos em suas superfícies. Estes resultados podem estar associados à aglomeração destas argilas no revestimento, de acordo com a elevada rugosidade superficial destas amostras (Tabela 2). Estes aglomerados podem atuar como promotores de defeitos, facilitando a passagem do eletrólito através do revestimento e, consequentemente, a corrosão do substrato.

Avaliou-se a migração subcutânea após 504 horas de exposição em névoa salina. A Tabela 3 apresenta os valores do desplacamento dos revestimentos estudados. Observa-se que as amostras contendo argila apresentaram um desplacamento médio semelhante ao da amostra sem argila (TE/0). No entanto, verificou-se que as amostras $\mathrm{TE} / 6 / 30 \mathrm{~B}$ e $\mathrm{TE} / 2 / 15 \mathrm{~A}$ apresentaram um desplacamento superior às demais.

\subsection{Espectroscopia de Impedância Eletroquímica (EIE)}

A Figura 2 apresenta os diagramas de Bode obtidos a partir análise de EIE em solução de $\mathrm{NaCl} 3,5 \%(\mathrm{~m} / \mathrm{v})$. módulo de impedância em baixas frequências medido durante EIS é um critério importante a ser considerado, pois quanto maior for o seu valor, melhor é a resistência à penetração de eletrólito e, como consequência, um melhor desempenho em atenuar a corrosão dos substratos [23].

Observa-se que todas as amostras apresentaram um excelente desempenho eletroquímico, uma vez que, após 1080 horas de imersão não se observou redução significativa nos valores de módulo de impedância em baixas frequências.

Para as amostras de tinta com argila MMT 30B, observa-se que a amostra com maior concentração (TE/6/30B) apresentou valor de impedância inferior às demais. Este resultado indica que um alto percentual de argila MMT 30B pode ter formado aglomerados e atuado como uma impureza, sensibilizando a estrutura da tinta e diminuindo a sua eficiência protetivo. Com isso, o eletrólito pode penetrar até o substrato por meio de pequenos defeitos, tais como microfissuras, propiciando a corrosão na interface metal/revestimento [2I]. Este resultado corrobora com os resultados de névoa salina (Figura I), onde a amostra TE/6/30B apresentou formação de corrosão vermelha intensa e elevado desplacamento (Tabela 3), quando comparado as demais amostras. Estes resultados podem estar associados à maior rugosidade superficial desta amostra, conforme relatado na Tabela 2, provocada por possíveis aglomerações da argila. 
Tabela 3. Medidas de desplacamento mínimo e máximo dos revestimentos de tinta em pó base epóxi após 504 horas de exposição à névoa salina

\begin{tabular}{cc}
\hline Amostra & Desplacamento Máximo $(\mathbf{m m})$ \\
\hline $\mathrm{TE} / 0$ & $\mathrm{I}, 2 \pm 0,3$ \\
$\mathrm{TE} / 2$ / 30B & $\mathrm{I}, 2 \pm 0,3$ \\
$\mathrm{TE} / 4$ / 30B & $\mathrm{I}, \mathrm{I} \pm 0,3$ \\
$\mathrm{TE} / 6$ / 30B & $2,8 \pm 0,3$ \\
$\mathrm{TE} / 2$ / I5A & $\mathrm{I}, 5 \pm 0,3$ \\
$\mathrm{TE} / 4$ / I5A & $\mathrm{I}, 6 \pm 0,3$ \\
$\mathrm{TE} / 6$ / I5A & $2,5 \pm 0,3$ \\
$\mathrm{TE} / 2$ / Mica & $\mathrm{I}, 8 \pm 0,3$ \\
$\mathrm{TE} / 4$ / Mica & $\mathrm{I}, 6 \pm 0,3$ \\
$\mathrm{TE} / 6$ / Mica & $\mathrm{I}, 7 \pm 0,3$ \\
\hline
\end{tabular}

Para as amostras contendo argila MMT I5A e mica moscovita, considerando-se todas as concentrações estudadas, observou-se um excelente desempenho protetivo, mesmo longos tempos de ensaio eletroquímico. Logo, o bom desempenho eletroquímico destas amostras denota que $\circ$ aumento da concentração de argila não interfere diretamente nas propriedades de barreira da tinta.

Para a tinta TE/0, este resultado confirma que a tinta em pó base epóxi apresenta uma excelente resistência à corrosão, o que já era esperado, por se tratar de uma tinta em pó termoconvertível que caracteriza-se pela ocorrência de reações químicas durante a cura, na qual transforma $\circ$ sistema de estrutura linear em um sistema de estrutura tridimensional, isto é, infusível, com alta resistência a solvente e a corrosão [10]. As resinas epoxídicas são caracterizadas pelo grupo epóxi, também chamado grupo glicidila que, está
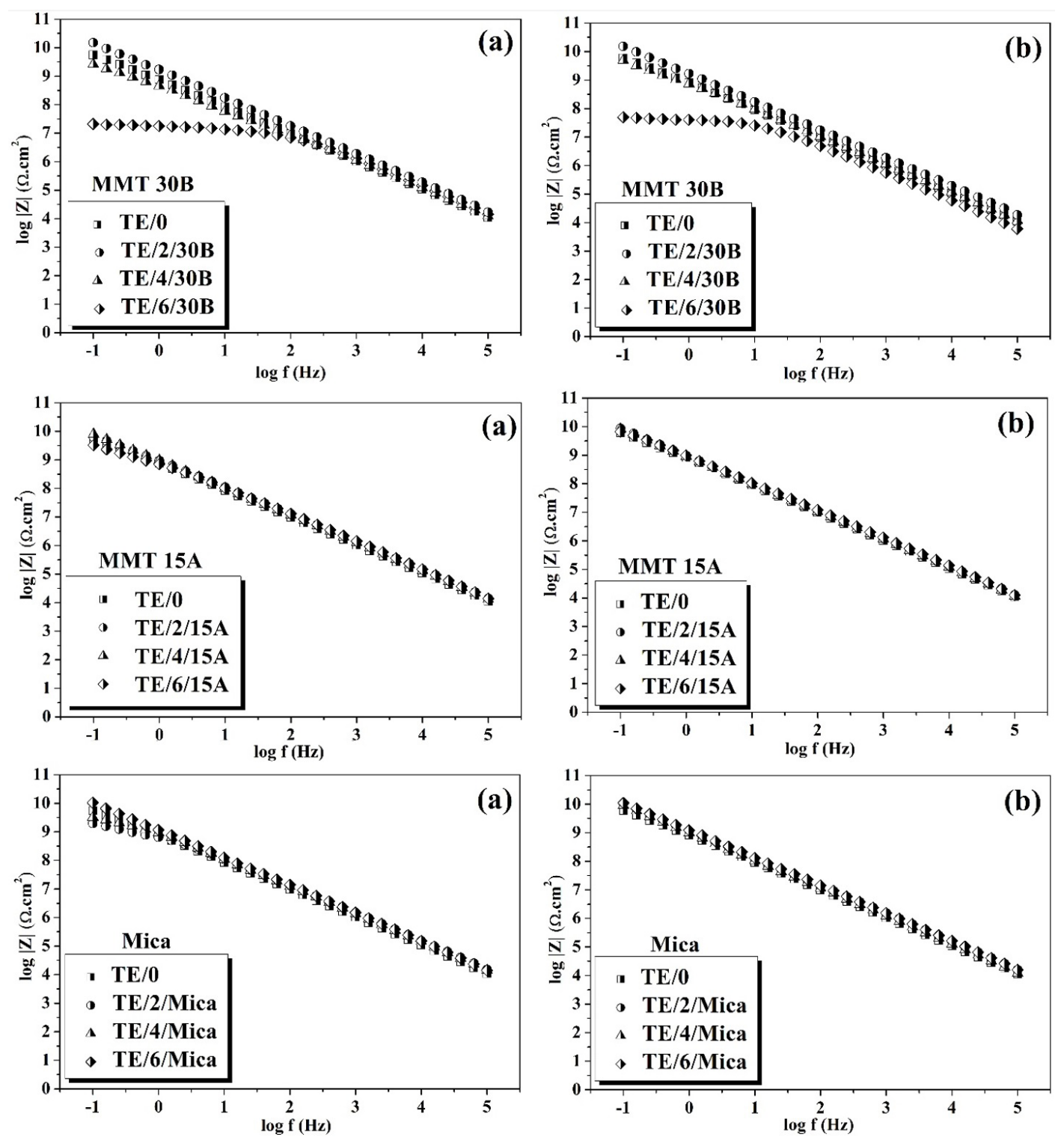

Figura 2. Diagramas de Bode dos revestimentos de tinta base resina epóxi contendo diferentes teores da argila mineral aplicados sobre aço carbono após (a) 240 horas de imersão e (b) 1080 horas imersão. 
localizado nos terminais da molécula linear. Este grupo, bem como os grupos hidroxila existentes na mesma molécula, permitem uma grande densidade de ligações reticulares durante o processo de cura, resultando em revestimentos com excelente aderência, dureza e resistência química [24].

\section{CONCLUSÕES}

Neste trabalho, diferentes tipos de argilas foram adicionados em uma resina epóxi, a fim de se estudar a influência da concentração (2, 4 e 6\%) destas argilas nas propriedades do revestimento sobre o aço AISI 1008.

Os resultados mostraram que a adição de argila montmorilonita MMT 30B e $15 \mathrm{~A}$ e de mica moscovita à resina epóxi não alterou o caráter hidrofílico do revestimento, permanecendo com ângulo de contato médio em torno de $70^{\circ}$ para todas as amostras estudadas.

A rugosidade superficial dos revestimentos aumentou com o aumento da concentração de argila. Este aumento foi mais significativo para os revestimentos contendo $6 \%$ de argila MMT, tanto 30B quanto I5A. Este resultado indica uma aglomeração das partículas de argila no interior do revestimento, o que promove um desnivelamento topográfico da superfície.
Quanto ao caráter protetivo, tanto na análise de névoa salina quanto na espectroscopia de impedância eletroquímica, todas as amostras apresentaram um excelente desempenho, atuando como barreira física e protegendo o substrato do ataque corrosivo. Contudo, a amostra contendo $6 \%$ de argila MMT 30B apresentou um desempenho protetivo inferior, quando comparado com as demais amostras estudadas. Este resultado pode estar associado a presença de aglomerados de argila no revestimento, que promove a formação de defeitos na superfície, facilitando a permeação do eletrólito e, por consequência, a corrosão do substrato metálico. Além disso, destaca-se também que a adição de $2 \%$ de argila MMT de ambos os tipos promoveu um menor desplacamento e menos formação de produtos de corrosão. Já a adição de Mica não promoveu melhorias significativas, independente da concentração.

Diante dos resultados apresentados, pode-se concluir que a adição de argilas montmorilonita à resina epóxi promoveu a formação de revestimentos nanocompósitos protetivos eficientes. Pequenas concentrações destas argilas aumentou a resistência à corrosão da tinta em pó. Contudo, concentrações superiores à $4 \%$ de MMT não são indicadas, pois podem promover a formação de aglomerados, diminuindo assim a eficiência protetiva destes revestimentos.

\section{REFERÊNCIAS}

I Piazza D, Lorandi NP, Pasqual Cl, Scienza LC, Zatteraa AJ. Influence of a microcomposite and a nanocomposite on the properties of an epoxy-based powder coating. Materials Science and Engineering A. 20I I;528:6769-6775.

2 Piazza D, Silveira DS, Lorandi NP, Birriel EJ, Scienza LC, Zattera AJ. Polyester-based powder coatings with montmorillonite nanoparticles applied on carbon steel. Progress in Organic Coatings. 20I 2;73:42-46.

3 Thostenson ET, Li C, Chou T-W. Nanocomposites in context. Composites Science and Technology. 2005;65:49I516.

4 Jordan J, Jacob KI, Tannenbaum R, Sharaf MA, Jasiuk I. Experimental trends in polymer nanocomposites - a review. Materials Science and Engineering A. 2005;393: I-II.

5 Djebara Y, El Moumen A, Kanit T, Madani S, Imad A. Modeling of the effect of particles size, particles distribution and particles number on mechanical properties of polymer-clay nanocomposites: Numerical homogenization versus experimental results. Composites. Part B, Engineering. 2016;86:135-142.

6 Yeh J-M, Chang K-C. Polymer/layered silicate nanocomposite anticorrosive coatings. Journal of Industrial and Engineering Chemistry. 2008; 14:275-29I.

7 Nunes MABS, Galvão LS, Ferreira TPM, Luiz EJFT, Bastos YLM, Santos ASF. Reprocessability of high impact polystyrene/clay nanocomposites in extrusion. Polymer Degradation \& Stability. 2016;125:87-96.

8 Chen C, Khobaid M, Curliss D. Epoxy layered-silicate nanocomposites. Progress in Organic Coatings. 2003;47:376-383.

9 Paul DR, Robeson LM. Polymer nanotechnology: Nanocomposites. Polymer. 2008;49:3 187-3204.

10 Hang TTX, Truc TA, Nam TH, Oanh VK, Jorcin JB, Pébère N. Corrosion protection of carbon steel by an epoxy resin containing organically modified clay. Surface and Coatings Technology. 2007;201:7408-74I 5.

II Carrasco F, Pagès P. Thermal degradation and stability of epoxy nanocomposites: Influence of montmorillonite content and cure temperature. Polymer Degradation \& Stability. 2008;93: 1000- 1007.

12 Chang K-C, Chen S-T, Lin H-F, Lin C-Y, Huang H-H, Yeh J-M, et al. Effect of clay on the corrosion protection efficiency of PMMA/Na +-MMT clay nanocomposite coatings evaluated by electrochemical measurements. European Polymer Journal. 2008;44:13-23. 
Ferreira et al.

I3 Burgentzlé D, Duchet J, Gérard JF, Jupin A, Fillon B. Solvent-based nanocomposite coatings. Journal of Colloid and Interface Science. 2004;278:26-39.

14 Cao X, Lee LJ, Widya T, Macosko C. Polyurethane/clay nanocomposites foams: processing, structure and properties. Polymer. 2005;46:775-783.

15 Stretz HA, Paul DR, Li R, Keskkula H, Cassidy PE. Intercalation and exfoliation relationships in melt-processed poly (styrene-co-acrylonitrile)/montmorillonite nanocomposites. Polymer. 2005;46(8):262I-2637.

16 Pandey JK, Reddy KR, Kumar AP, Singh RP. An overview on the degradability of polymer nanocomposites. Polymer Degradation \& Stability. 2005;88:234-250.

17 Saarivirta EH, Vaganov GV, Yudin VE, Vuorinen J. Characterization and corrosion protection properties of epoxy powder coatings containing nanoclays. Progress in Organic Coatings. 2013;76:757-767.

I8 Wang K, Wang L, Wu J, Chen L, He C. Preparation of highly expholiated epoxy/clay nanocomposites by "slurry compounding": process and mechanisms. Langmuir. 2005;21:36/3-3618.

19 Shi X, Nguyen TA, Suo Z, Liu Y, Avci R. Effect of nanoparticles on the anticorrosion and mechanical properties of epoxy coating. Surface and Coatings Technology. 2009;204:237-245.

20 Joanny JF, Gennes PGJ. A model for contact angle hysteresis. Chemical Physics. 1984;81:552.

2I Suegama PH, Fugivara CS, Benedetti AV, Delgado J, Guilemany JM. Electrochemical characterisation study of coatings obtained by High Velocity Oxy-Fuel Sprayin (HVOF). Portugaliae Electrochimica Acta. 2003;21:14I-I54.

22 Bagherzadeh MR, Mousavinejad T. Preparation and investigation of anticorrosion properties of water-based epoxyclay nanocoating modified by $\mathrm{Na}+-\mathrm{MMT}$ and Cloisite 30B. Progress in Organic Coatings. 20I 2;74:589-595.

23 Warner TJ, Schmidt MP, Sommer F, Bellot D. Characterization of corrosion initiation on 2024 aluminium alloy by atomic force microscopy. Zeitschrift fur Metallkunde. 1995;86(7):494-50I.

24 Darmiani E, Danaee I, Rashed GR, Zaarei D. Formulation and study of corrosion prevention behavior of epoxy cerium nitrate - montmorillonite nanocomposite coated carbon steel. Journal of Coatings Technology and Research, American Coatings Association \& Oiland Colour Chemists Association. 2013;10(4):493-502.

Recebido em: 16 Jun. 2017

Aceito em: II Jul. 2018 